Supporting Information

\title{
Theoretical Investigation of the Structure-Property Correlation of MXenes as Anode Materials for Alkali Metal Ion Batteries
}

Na Li ${ }^{a}$ Yiran Li, ${ }^{b}$ Xiaohong Zhu, ${ }^{a}$ Changxiong Huang ${ }^{a}$ and Jun Fan ${ }^{a, c^{*}}$

${ }^{a}$ Department of Materials Science\& Engineering, City University of Hong Kong, Hong Kong, China

${ }^{b}$ Department of Mechanical Engineering, City University of Hong Kong, Hong Kong, China

${ }^{c}$ Center for Advance Nuclear Safety and Sustainable Development, City University of Hong Kong, Hong Kong, China

*Corresponding author: junfan@cityu.edu.hk 
Table S1. The optimized lattice constants $(a)$ and the average distance $\left(d_{(\mathrm{Li}-\mathrm{O})}, d_{(\mathrm{Na}-\mathrm{O})}\right.$ and $\left.d_{(\mathrm{K}-\mathrm{O})}\right)$ between single $\mathrm{Li}, \mathrm{Na}$ and $\mathrm{K}$ atom and the closest oxygen functional groups of $\mathrm{M}_{3} \mathrm{C}_{2} \mathrm{O}_{2}$ in the adsorption configuration.

\begin{tabular}{ccccc}
\hline $\mathbf{M}_{3} \mathbf{C}_{2} \mathbf{O}_{2}$ & $\boldsymbol{a}, \AA$ & $\boldsymbol{d}_{(\mathbf{L i}-\mathbf{0})}, \AA$ & $\boldsymbol{d}_{(\mathbf{N a}-\mathbf{0})}, \AA$ & $\left.\boldsymbol{d}_{(\mathbf{K}-\mathbf{0})}\right), \AA$ \\
\hline $\mathrm{V}_{3} \mathrm{C}_{2} \mathrm{O}_{2}$ & 2.91 & 1.955 & 2.297 & 2.634 \\
$\mathrm{Ti}_{3} \mathrm{C}_{2} \mathrm{O}_{2}$ & 3.05 & 1.996 & 2.309 & 2.648 \\
$\mathrm{Nb}_{3} \mathrm{C}_{2} \mathrm{O}_{2}$ & 3.16 & 2.007 & 2.333 & 2.656 \\
$\mathrm{Hf}_{3} \mathrm{C}_{2} \mathrm{O}_{2}$ & 3.27 & 2.044 & 2.368 & 2.693 \\
$\mathrm{Zr}_{3} \mathrm{C}_{2} \mathrm{O}_{2}$ & 3.31 & 2.049 & 2.393 & 2.713 \\
\hline
\end{tabular}

Table S2. The optimized lattice constants $(a)$ and the measured average distances between Li-O, Li-Li, Na-O, Na-Na, K-O and K-K atoms of monolayer $\mathrm{Li}$, Na and $\mathrm{K}$ atoms on five $\mathrm{M}_{3} \mathrm{C}_{2} \mathrm{O}_{2}(\mathrm{M}=\mathrm{V}$, $\mathrm{Ti}, \mathrm{Nb}$, Hf and $\mathrm{Zr}$ ).

\begin{tabular}{|c|c|c|c|c|c|c|c|}
\hline \multirow[t]{2}{*}{$\mathbf{M}_{3} C_{2} O_{2}$} & \multirow[t]{2}{*}{$a, \AA$} & \multicolumn{2}{|c|}{$1^{\text {st }}$ layer Li } & \multicolumn{2}{|c|}{$1^{\text {st }}$ layer Na } & \multicolumn{2}{|c|}{$1^{\text {st }}$ layer K } \\
\hline & & $d_{(\mathrm{Li}-\mathbf{O})}, \AA$ & $d_{(\mathrm{Li}-\mathrm{Li})}, \AA$ & $d_{(\mathrm{Na}-\mathrm{O})}, \AA$ & $\boldsymbol{d}_{(\mathrm{Na}-\mathrm{Na})}, \AA$ & $d_{(\mathrm{K}-\mathrm{O})}, \AA$ & $d_{(\mathrm{K}-\mathrm{K})}, \AA$ \\
\hline $\mathrm{V}_{3} \mathrm{C}_{2} \mathrm{O}_{2}$ & 2.91 & 1.864 & 2.910 & 2.280 & 2.910 & 2.450 & 2.910 \\
\hline $\mathrm{Ti}_{3} \mathrm{C}_{2} \mathrm{O}_{2}$ & 3.05 & 1.911 & 3.050 & 2.323 & 3.050 & 2.506 & 3.050 \\
\hline $\mathrm{Nb}_{3} \mathrm{C}_{2} \mathrm{O}_{2}$ & 3.16 & 1.928 & 3.160 & 2.359 & 3.160 & 2.552 & 3.160 \\
\hline $\mathrm{Hf}_{3} \mathrm{C}_{2} \mathrm{O}_{2}$ & 3.27 & 2.044 & 3.270 & 2.400 & 3.270 & 2.616 & 3.270 \\
\hline $\mathrm{Zr}_{3} \mathrm{C}_{2} \mathrm{O}_{2}$ & 3.31 & 2.048 & 3.310 & 2.415 & 3.310 & 2.624 & 3.310 \\
\hline
\end{tabular}



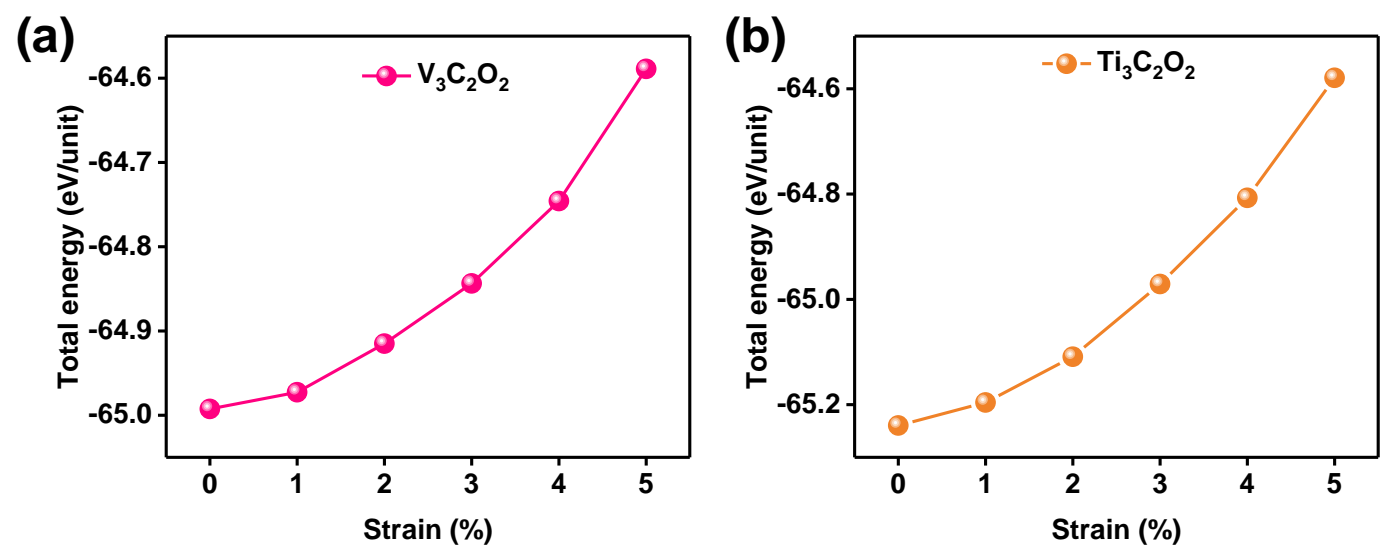

Figure S1. Strain dependence of total energies for $\mathrm{V}_{3} \mathrm{C}_{2} \mathrm{O}_{2}$ (a) and $\mathrm{Ti}_{3} \mathrm{C}_{2} \mathrm{O}_{2}$ (b).
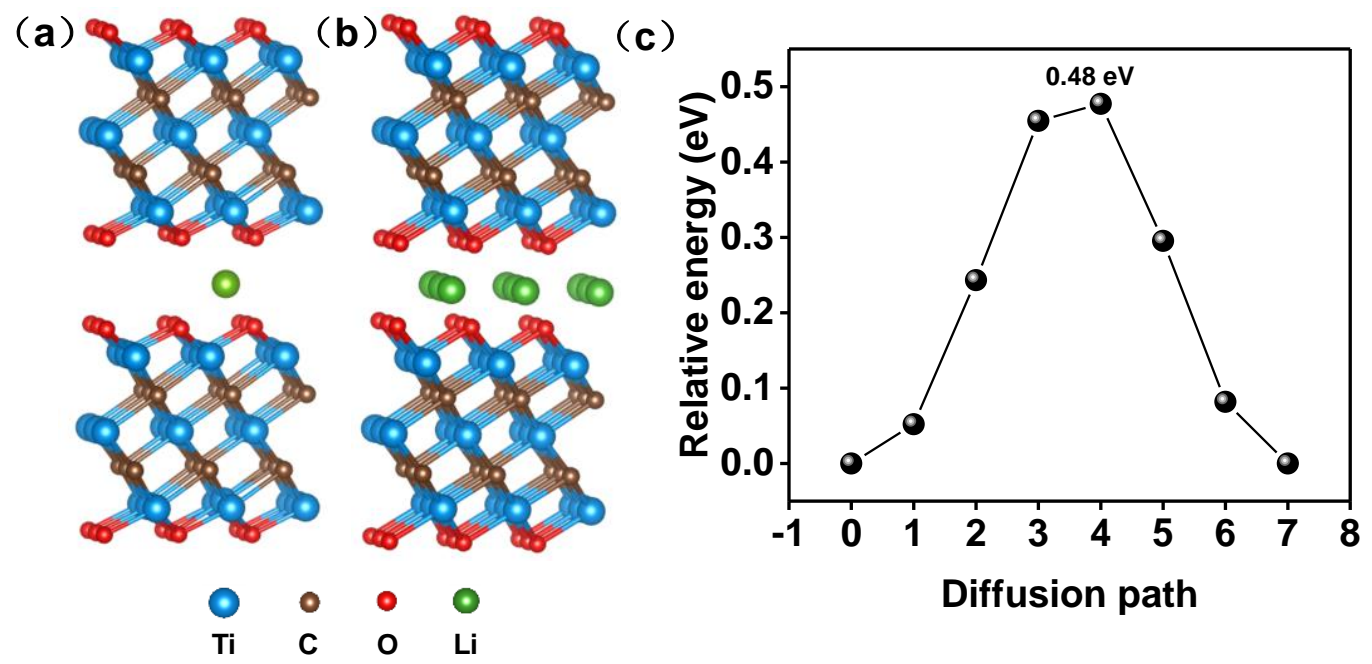

Figure S2. Side views of single Li atom (a) and monolayer Li atoms (b) as well as the diffusion barrier profile of $\mathrm{Li}$ atom on the interlayer of bilayer $\mathrm{Ti}_{3} \mathrm{C}_{2} \mathrm{O}_{2}$.

Table S3. The adsorption energy of single Li atom and Li atom layer as well as the diffusion energy barrier on monolayer and bilayer $\mathrm{Ti}_{3} \mathrm{C}_{2} \mathrm{O}_{2}$.

\begin{tabular}{cccc}
\hline $\mathrm{Ti}_{3} \mathrm{C}_{2} \mathrm{O}_{2}$ & $\begin{array}{c}E_{\text {ads }}(\text { single Li } \\
\text { atom })(\mathrm{eV} / \text { atom })\end{array}$ & $\begin{array}{c}E_{\text {ads }}(\mathrm{Li} \text { atom } \\
\text { layer })(\mathrm{eV} / \text { atom })\end{array}$ & $\begin{array}{c}\text { Diffusion energy } \\
\text { barrier }(\mathrm{eV})\end{array}$ \\
\hline $\begin{array}{c}\text { Monolayer } \\
\text { Bilayer }\end{array}$ & -2.32 & -1.45 & 0.37 \\
\hline
\end{tabular}

